\title{
A Systematic Review of Intensive Cardiopulmonary Management after Spinal Cord Injury
}

\author{
Steven Casha and Sean Christie
}

\begin{abstract}
Intensive cardiopulmonary management is frequently undertaken in patients with spinal cord injury (SCI), particularly due to the occurrence of neurogenic shock and ventilatory insufficiency and in an attempt to reduce secondary injury. We undertook a systematic review of the literature to examine the evidence that intensive care management improves outcome after SCI and to attempt to define key parameters for cardiopulmonary support/ resuscitation. We review the literature in five areas: management of SCI patients in specialized centers, risk in SCI patients of cardiopulmonary complications, parameters for blood pressure and oxygenation/ventilation support following SCI, risk factors for cardiopulmonary insufficiency requiring ICU care after SCI, and preventative strategies to reduce the risks of cardiopulmonary complications in SCI patients. The literature supports that, in light of the significant incidence of cardiorespiratory complications, SCI patients should be managed in a monitored special care unit. There is weak evidence supporting the maintenance of MAP $>85 \mathrm{mmHg}$ for a period extending up to 1 week following SCI.
\end{abstract}

Key words: intensive care; nonsurgical management; spinal cord injury; systematic review

\section{Introduction}

$S^{\mathrm{c}}$ BJECTS PRESENTING WITH SPINAL CORD INJURY (SCI) are requently trauma patients and thus can exhibit multisystem injuries requiring intensive management. However, the consequences of the SCI itself are also capable of rendering patients critically ill. Key issues are the occurrence of neurogenic shock and the risk of ventilatory insufficiency in these patients. Furthermore, it has been argued that intensive care management of the SCI patient has likely had the greatest impact on improved outcomes over the past 20 years. Vascular and respiratory implications of both the SCI and other associated injuries contribute to secondary injury after SCI and worsen neurological outcome. However, hemodynamic support and ventilatory assistance are invasive protocols. They represent a double-edged sword; while likely improving outcome, they may also contribute to adverse events and may have implications for mortality. It thus appears that optimization of the parameters used in guiding intensive management has the potential for immediate impact on patient outcomes.

The aim of this systematic review was to examine the evidence that intensive care management improves outcome after spinal cord injury and to attempt to define key parameters for cardiopulmonary support/resuscitation.
Five specific questions were identified in order to address these objectives: (1) Do spinal cord injured patients warrant management in specialized centers with specific cardiopulmonary protocols and access to ICU care? (What is the incidence of significant cardiopulmonary adverse events, and does specialized care improve outcome and/or reduced complications?) (2) What is the time at risk for cardiopulmonary complications requiring ICU management availability? (3) What parameters for blood pressure and oxygenation/ ventilation over what time period areassociated with improved outcome? (4) Are there any risk factors that are predictive of the need for ICU management? (5) What preventative strategies can be undertaken to reduce the risks of cardiopulmonary complications requiring ICU care (excluding DVT prophylaxis, surgical management, and nutrition).

\section{Methods}

\section{Search strategy and selection criteria}

A systematic review of the online NIH Medline database (Pubmed) was conducted to identify all studies that address cardiovascular and respiratory management of spinal cord injury patients published between 1975 and 2008. The search was conducted by combining the term "spinal cord injury"

Division of Neurosurgery, Dalhousie University, Halifax, Nova Scotia, Canada. 
with "non-surgical management," "nonsurgical management," "intensive care," "acute" AND "cardiovascular," "acute," AND "respiratory" (518 articles). Additional articles were retrieved from the review articles identified.

The titles and abstracts of each of the citations retrieved through the search process were reviewed for potential inclusion. The original articles were retrieved if the abstract and title did not provide sufficient detail. All studies that addressed the cardiovascular and/or respiratory management of SCI patients were retrieved in full and reviewed and assessed for final inclusion.

\section{Inclusions and exclusions}

This review was limited to original articles written in English and published in peer-reviewed journals conducting human subject-level investigation. Included trials were limited to those in which either all or an identified subgroup of subjects had suffered from acute traumatic SCI (injury within 1 month). Non-English language studies, reviews, letters to the editor, commentaries, and preclinical studies were excluded. Case reports and reports with sample sizes of fewer than three were excluded. Articles that described pharmacological approaches to neuroprotection and surgical management were excluded.

\section{Data abstraction}

We abstracted the following information: study design, sample size, patient characteristics, details of the treatment administered or the cohort identified, and outcome observations. The abstracted data were compiled into evidentiary tables to address each question as follows:

1. Do spinal cord injured patients warrant management in specialized centers with specific cardiopulmonary protocols and access to ICU care? Evidence to address this question came from two sources:

a. Peer-reviewed literature that details the incidence of significant cardiopulmonary adverse events (25 articles).

b. Peer-reviewed literature that shows improved outcome and/or reduced complications with management in specialized centers or with specialized protocols (21 articles).

2. What is the time at risk for cardiopulmonary complications requiring ICU management availability?

Articles that describe timing of cardiopulmonary complications requiring ICU management or specify a risk period or treatment period start and/or stop (19 articles).

3. What parameters for blood pressure and oxygenation/ ventilation over what time period are associated with improved outcome?

Articles that specify a protocol for cardiopulmonary ICU management (eight articles).

4. Are there any risk factors that are predictive of the need for ICU management?

Articles that describe risk factors for cardiopulmonary complications leading to ICU admission or death (19 articles).

5. What preventative strategies can be undertaken to reduce the risks of cardiopulmonary complications requiring
ICU care (excluding DVT prophylaxis, surgical management, and nutrition)?

Articles describing strategies to decrease the incidence of cardiopulmonary complications requiring ICU admission and/or mortality (nine articles).

The articles reviewed are summarized in Table 1; questions addressed by each article are indicated.

\section{Results}

\section{Do spinal cord injured patients warrant management in specialized centers with specific cardiopulmonary protocols and access to ICU care?}

Evidence to address this question was gathered into two sources: literature that details the incidence of significant cardiopulmonary adverse events and literature that examines outcome and/or complications with management in specialized centers or with specialized protocols. These comprised 25 and 21 articles, respectively.

We found 19 case series, four cohort control studies, and two evaluations of diagnostic studies that described incidence of significant cardiopulmonary adverse events.

Regarding the incidence of cardiovascular insufficiency or serious adverse events, Vale and associates (1997) described the outcome of patients treated with a cardiopulmonary management protocol aiming to maintain MAP $>85$. They found that $90 \%$ of patients with cervical complete (ASIA A) SCI required pressor support. This incidence was less in incomplete SCI (52\%) and with thoracic SCI (31\%). Levi and associates (1993) similarly undertook a protocol to maintain MAP $>90$. They observed that $16 \%$ of patients had systolic blood pressure (SBp) $<90$ at admission and $82 \%$ exhibited volume-resistant hypotension within the first 7 days. Lehmann and colleagues (1987) examined the incidence of cardiovascular abnormalities among SCI patients admitted to the ICU within $12 \mathrm{~h}$ of injury. All cervical severe SCI patients experienced bradycardia. This was less frequent in milder cervical injury and in thoracic SCI (35 and 13\%, respectively). Similarly, hypotension, use of pressors, and cardiac arrest were only observed in cervical severe SCI $(68 \%, 35 \%$, and $16 \%$, respectively). Arrhythmia was also more frequent in this group. Piepmeier and colleagues (1985) described a series of 45 cervical SCI patients. These authors aimed to maintain SBp $>100$. They found that most patients' hypotension could be treated with fluid therapy; however 9 of 45 required vasopressor therapy. They noted a correlation between severity of injury and incidence and severity of cardiovascular irregularities. Gschaedler and co-workers (1979) found that $40 \%$ of the acute SCI patients they studied exhibited multi-organ system injury. They observed that, of the $42 \%$ mortality seen in their case series, 10 were due to cardiovascular failure. These studies vary in several ways but most notably in the population described and in the definition of hemodynamic compromise. Nonetheless, they all indicate that a significant number of SCI patients require cardiovascular support with fluids and vasopressor therapy and that the incidence of these events is correlated with severity of injury and is higher with cervical SCI and when hypotension is present at admission.

The incidence of pulmonary insufficiency requiring ventilatory support is also presented in several reports. Reines and Harris (1987) found $35.7 \%$ of 123 acute SCI patients experi- 


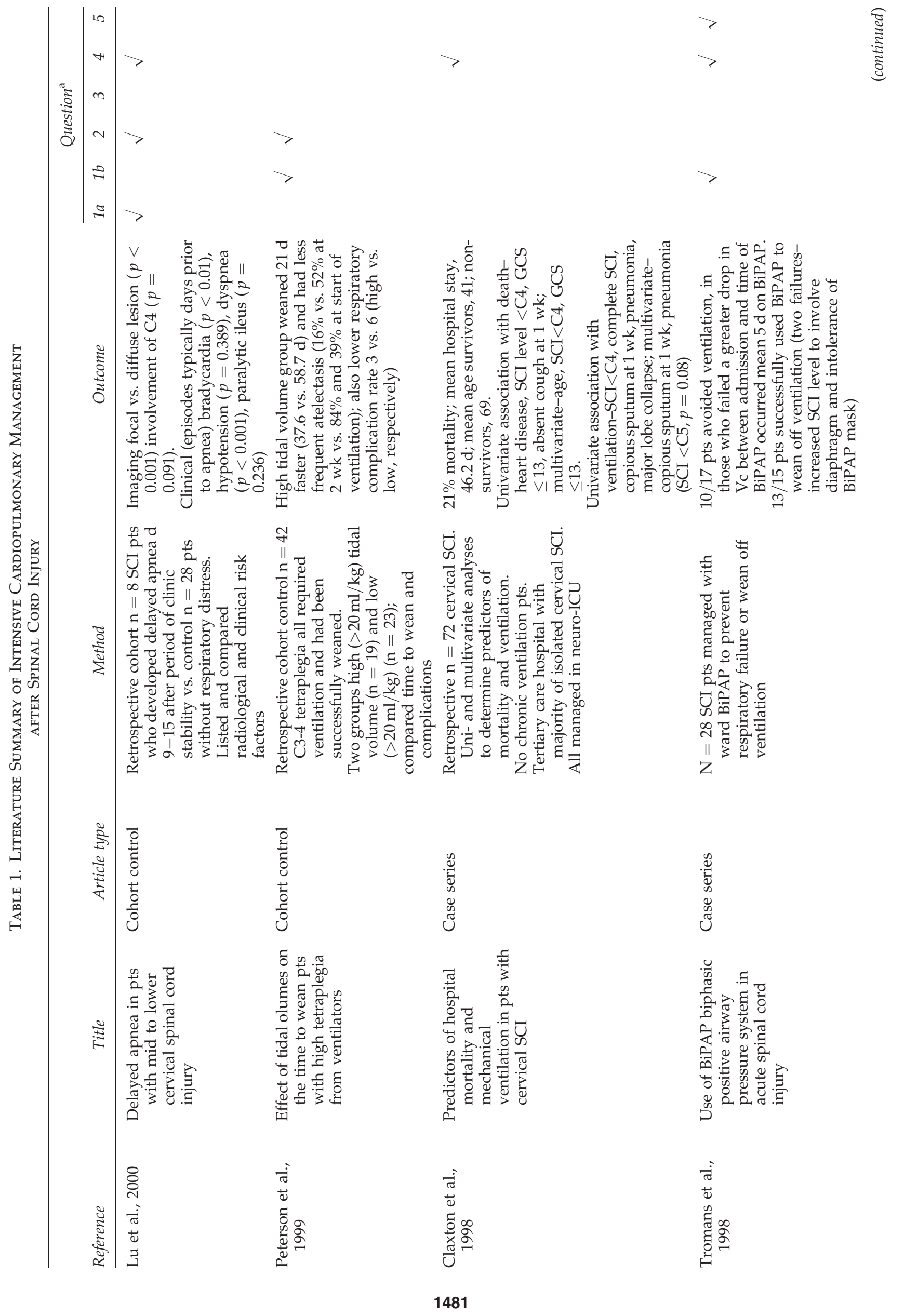




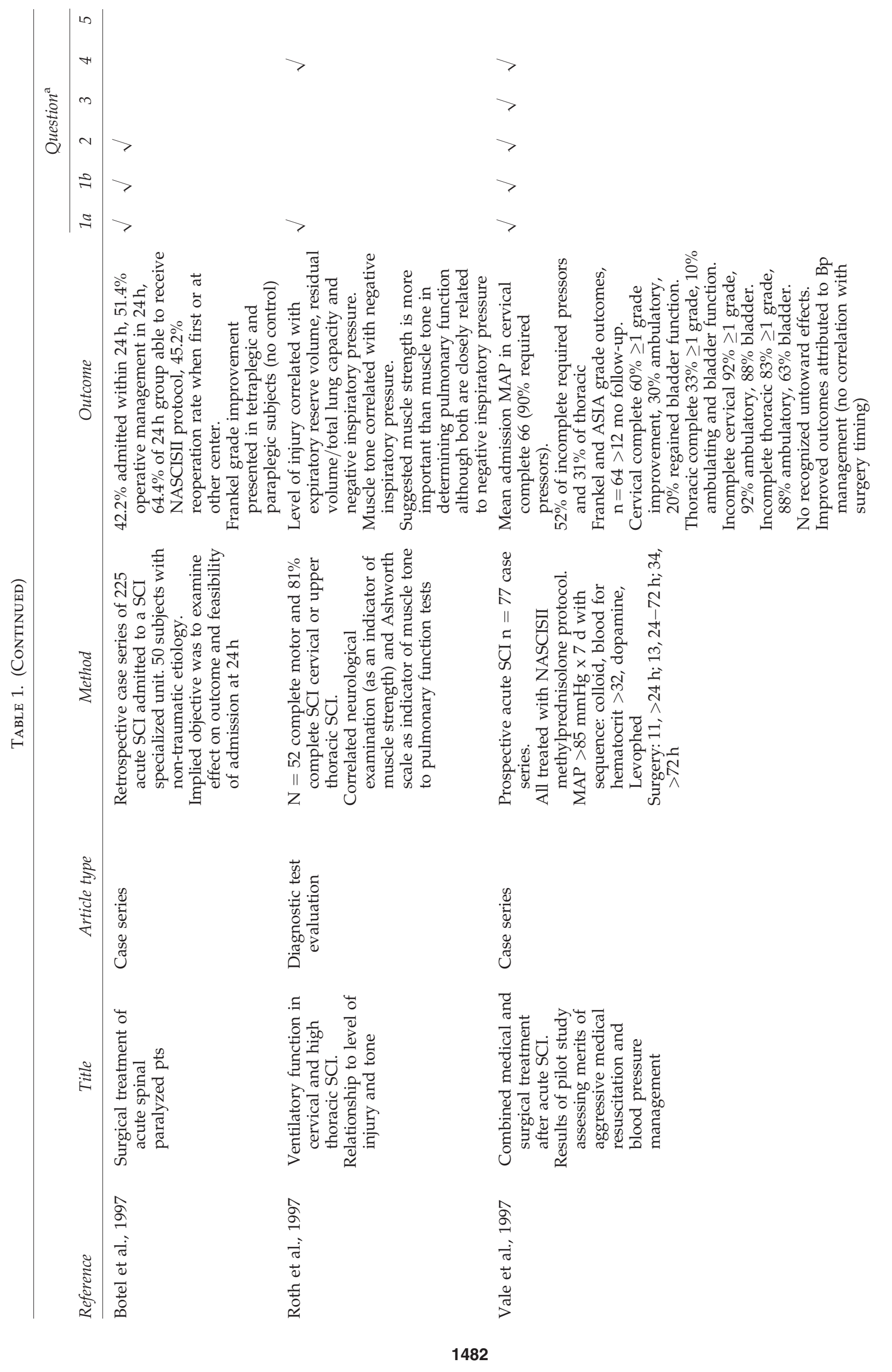



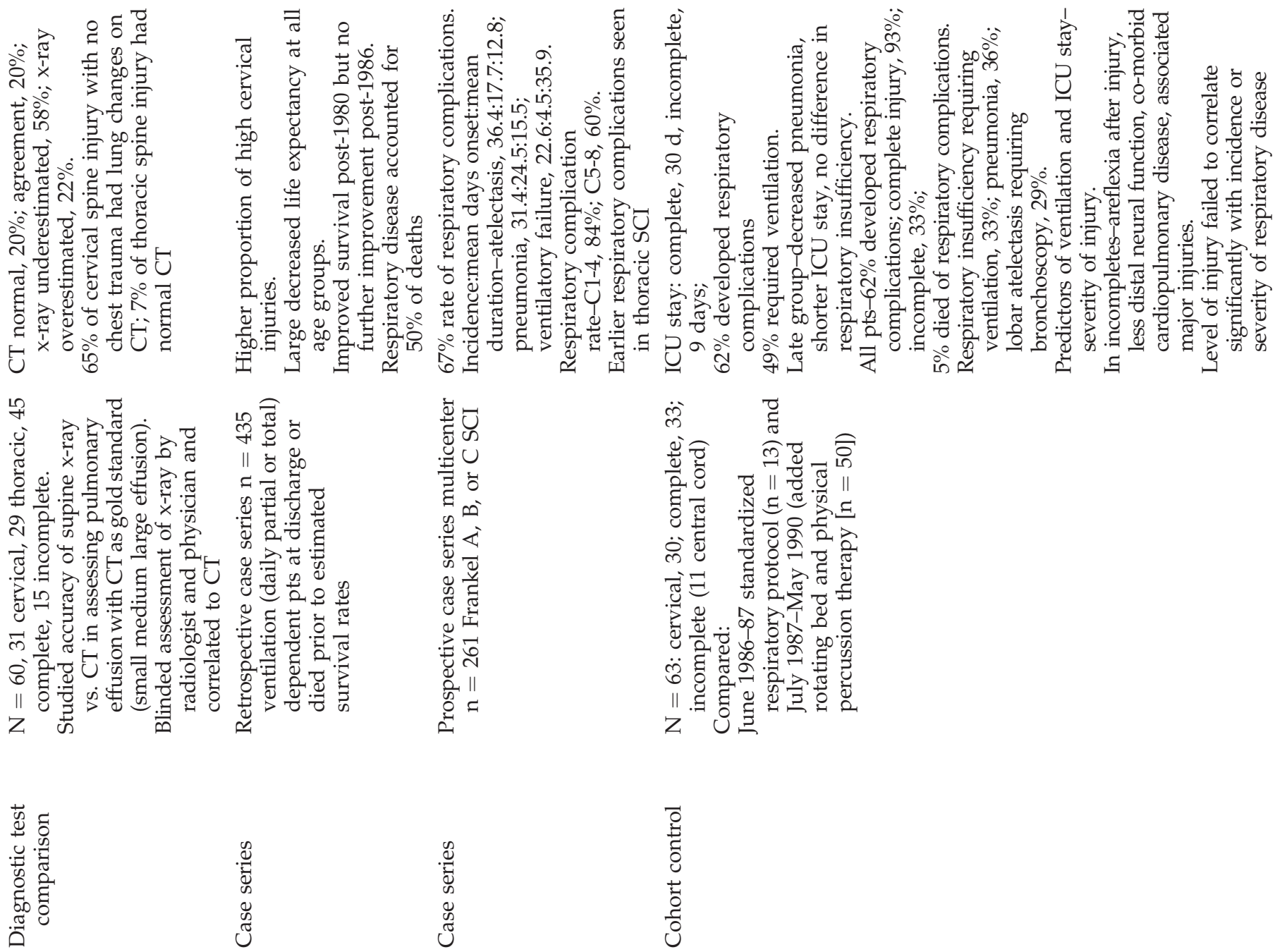

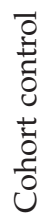
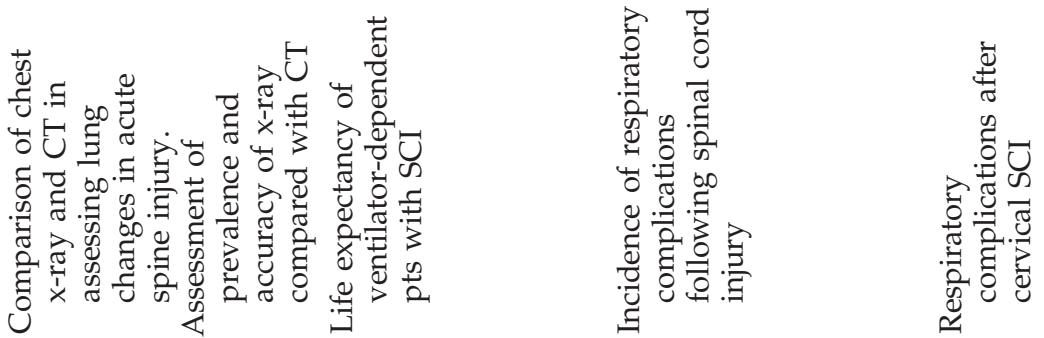

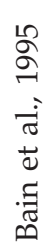
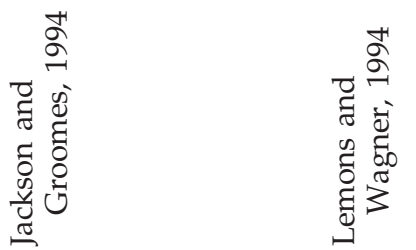


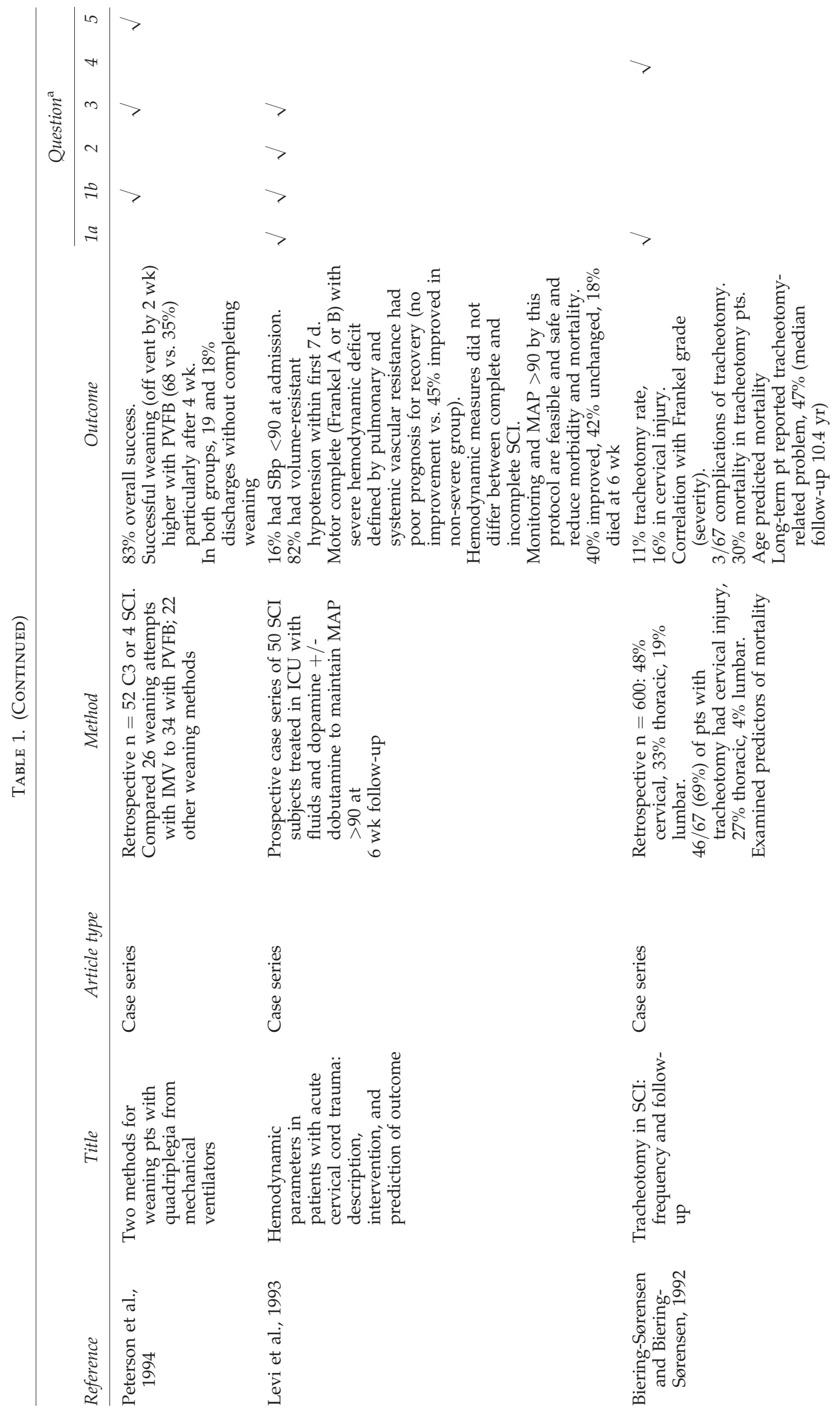



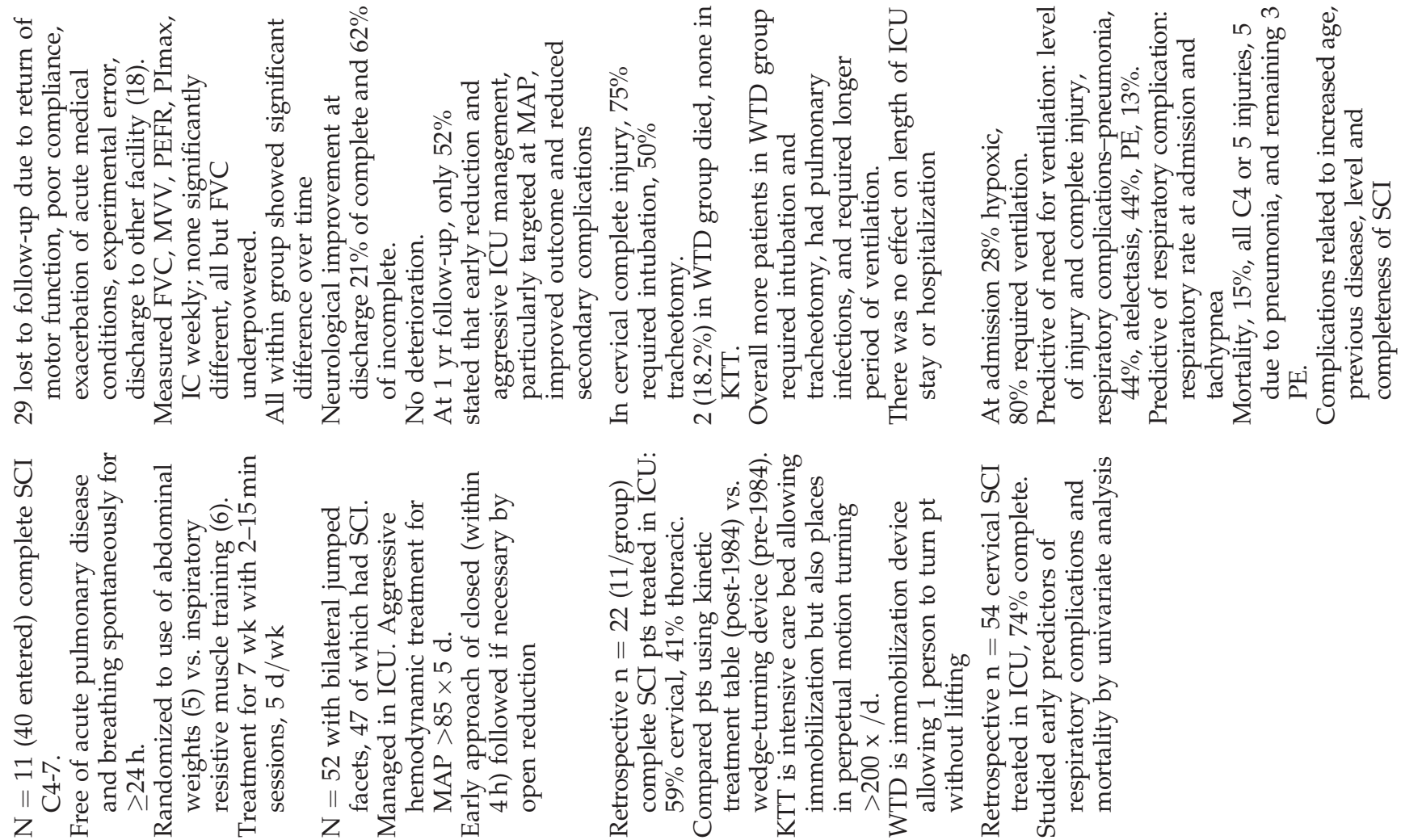

讯

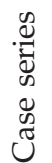

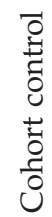
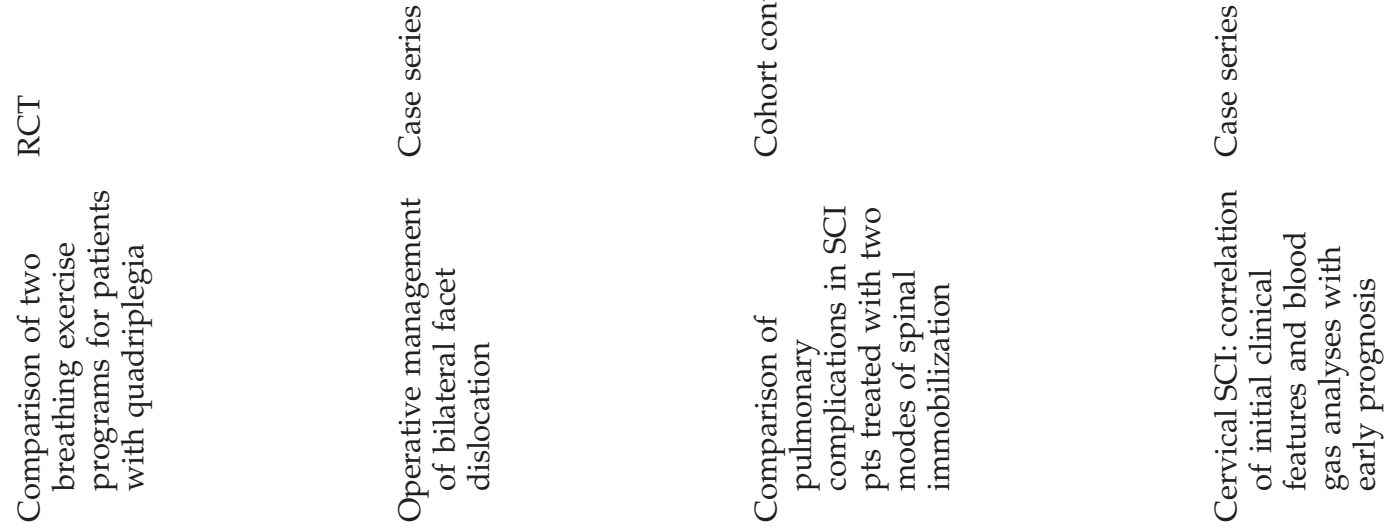

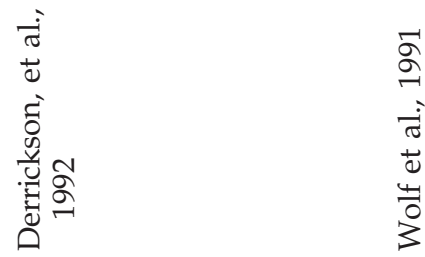

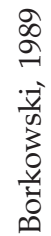

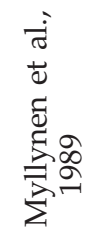




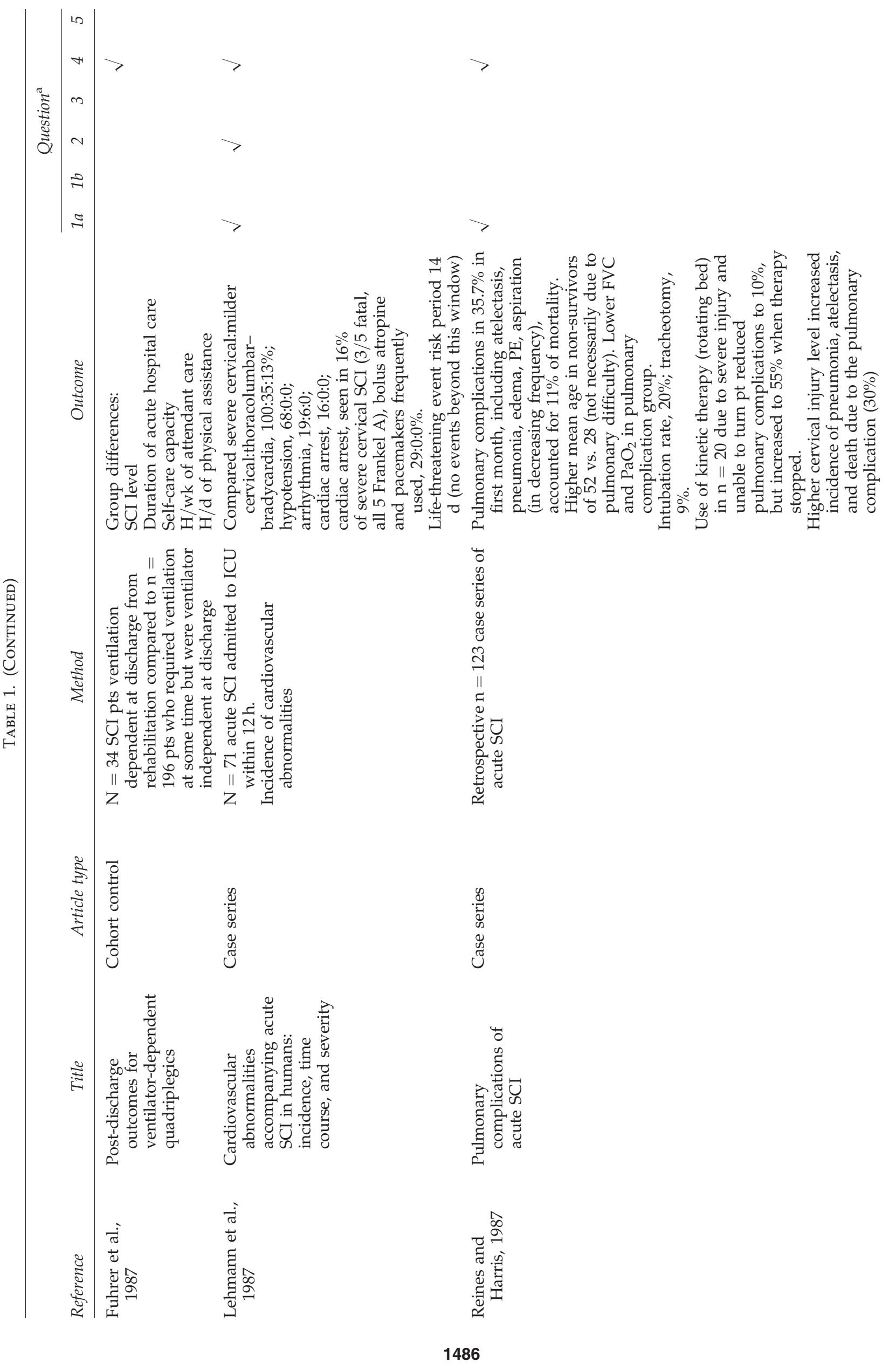




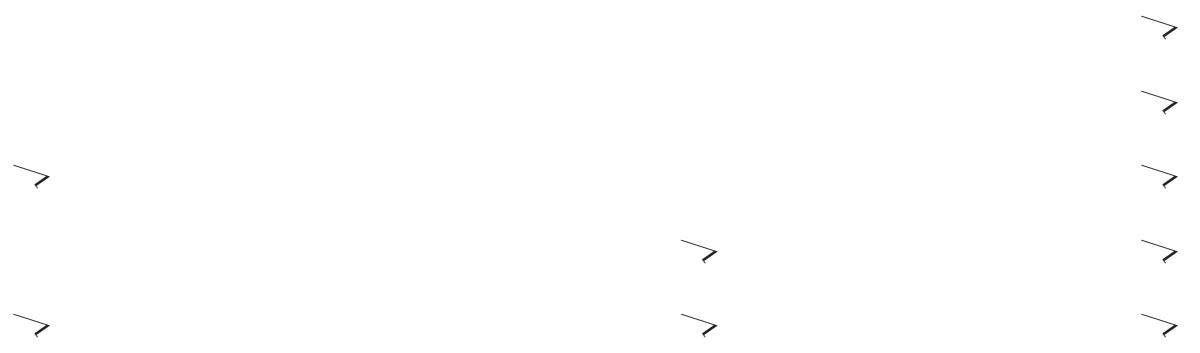

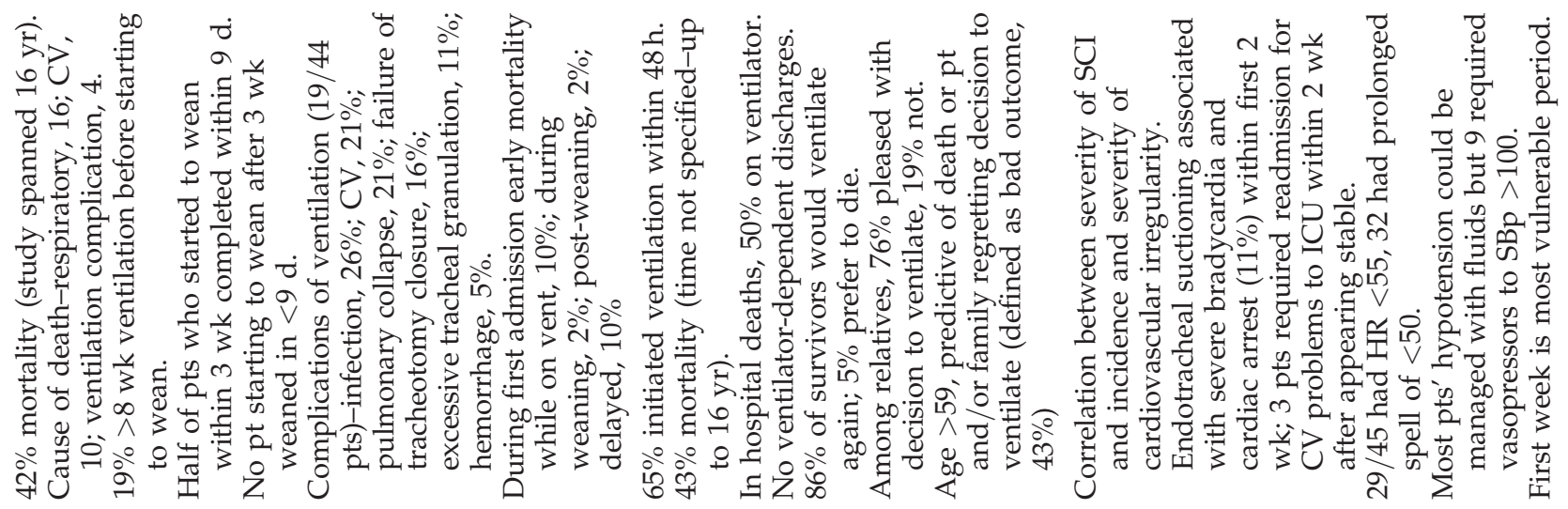
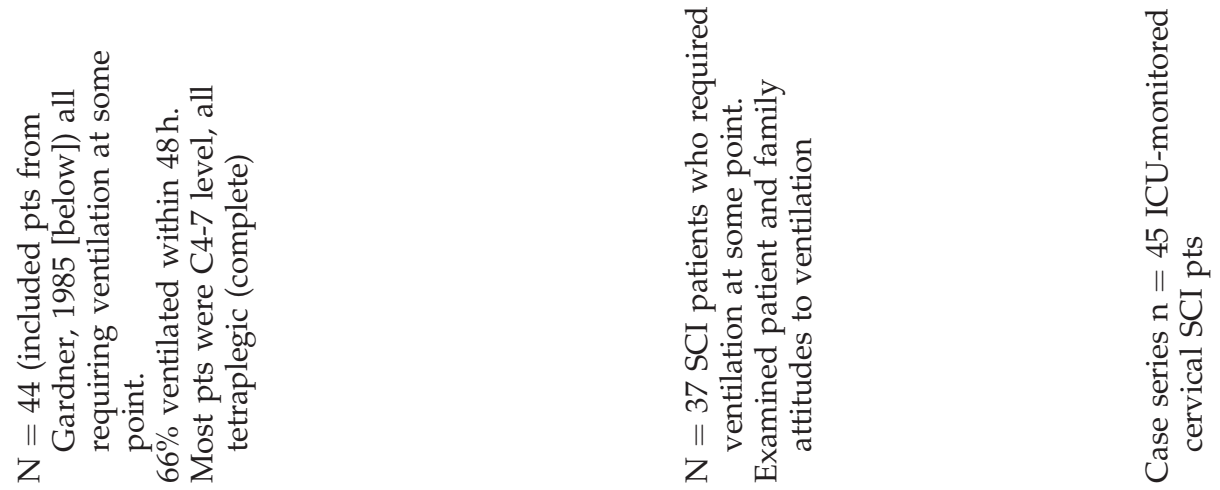

$\mathscr{0}$
$\stackrel{0}{0}$
0
0
$\tilde{J}$
$\tilde{J}$
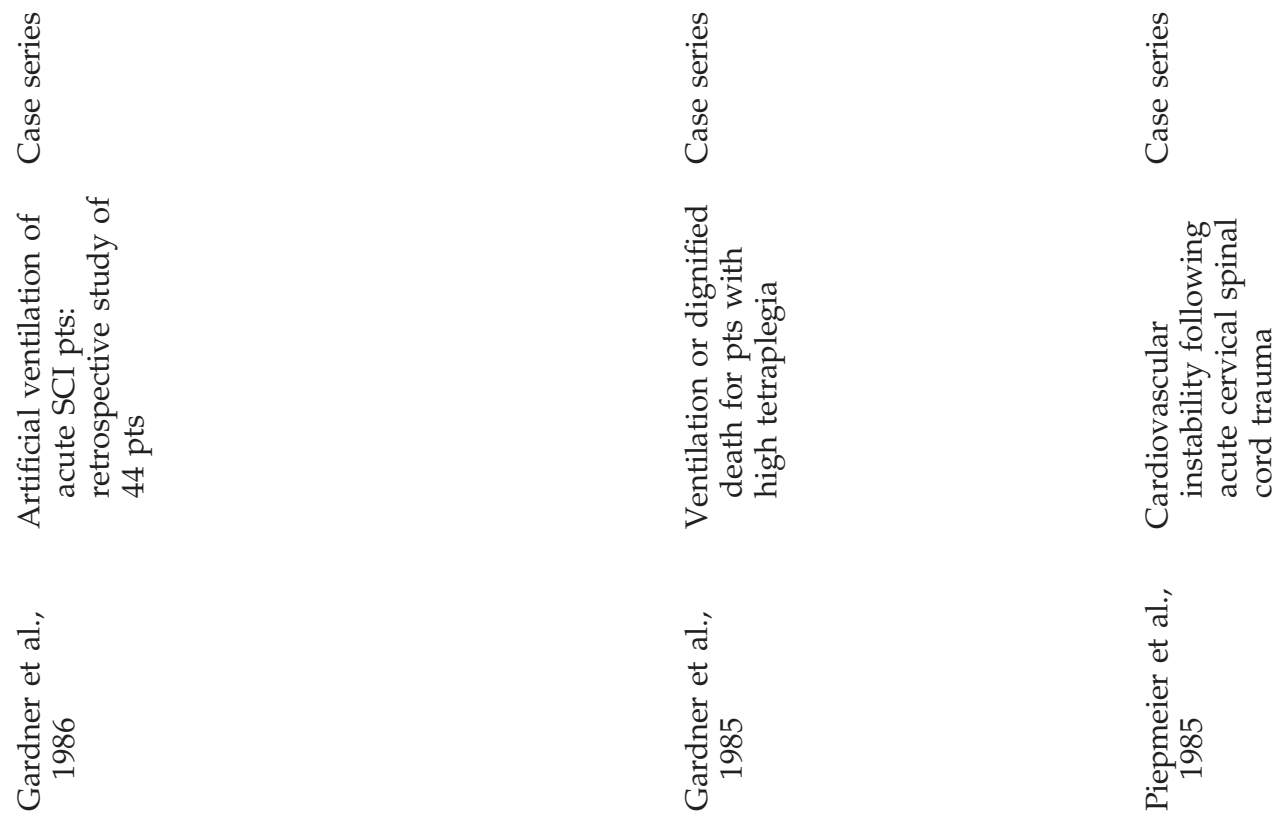


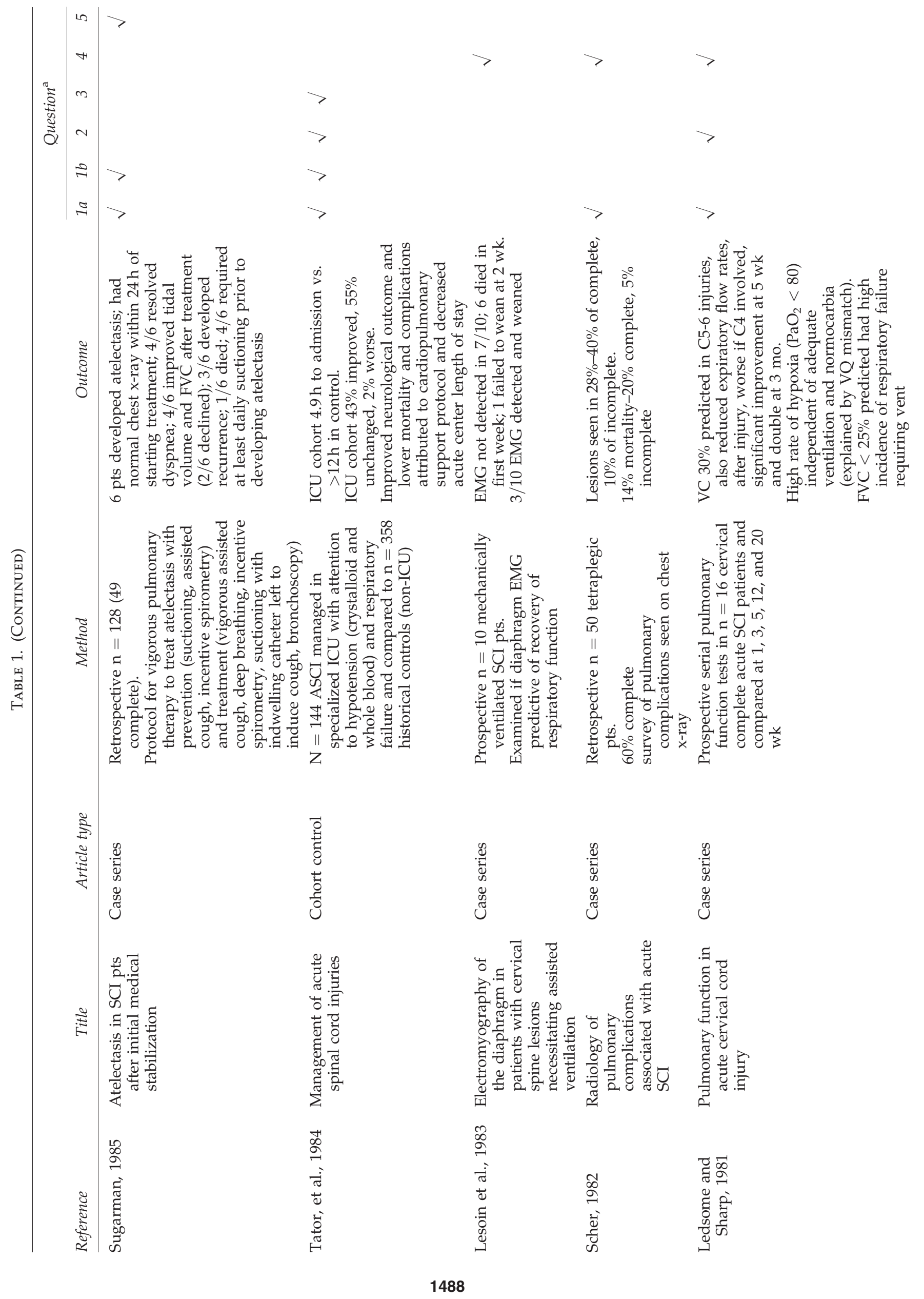



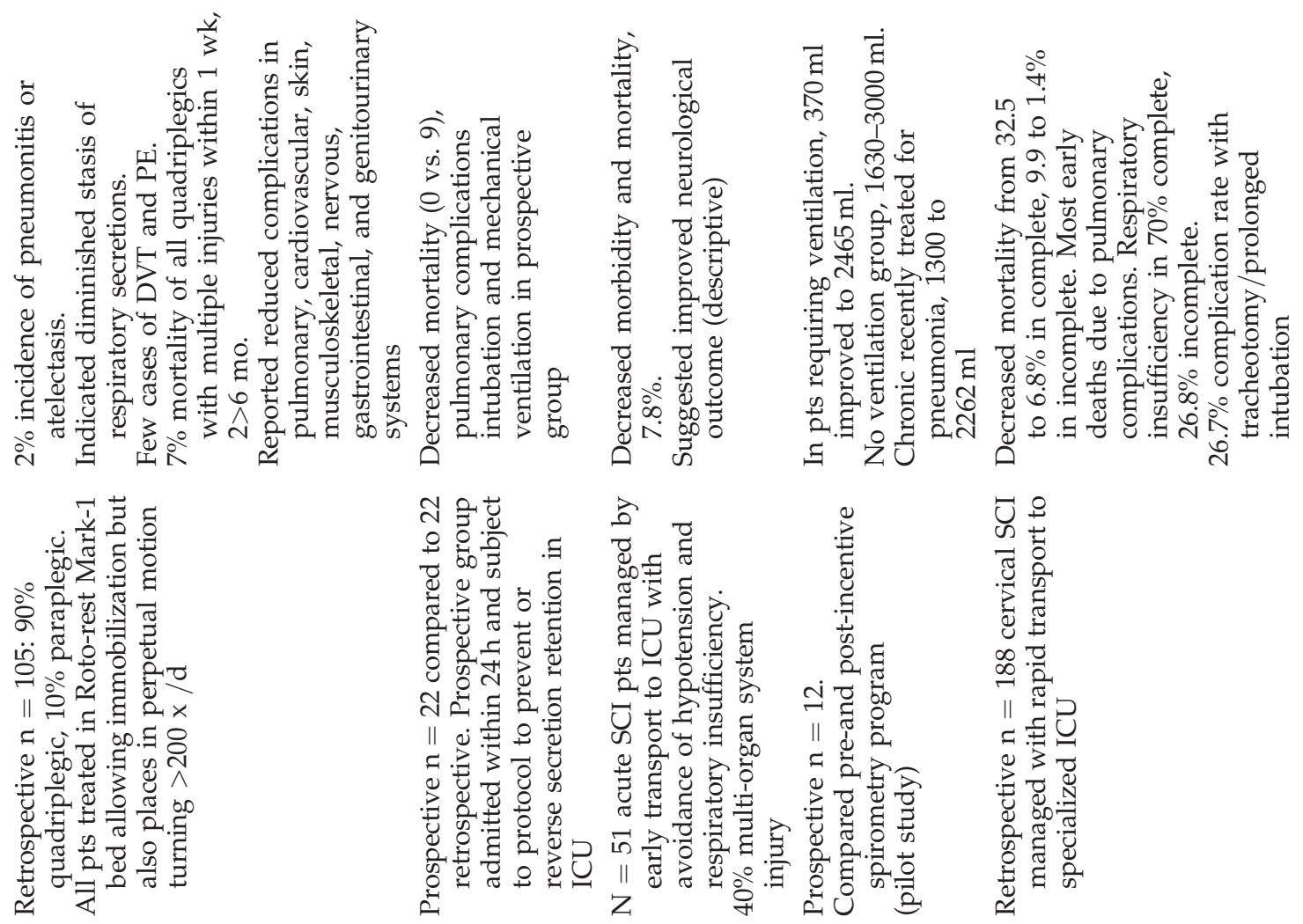

$\mathscr{0}$
$\stackrel{0}{0}$
0
0
$\tilde{J}$
$\tilde{J}$

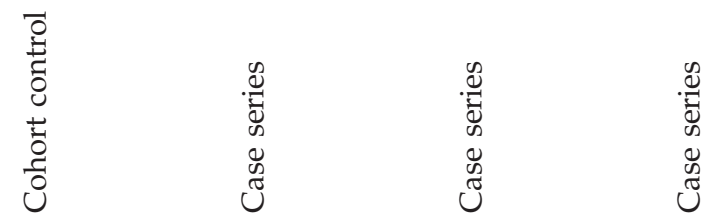

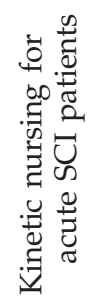

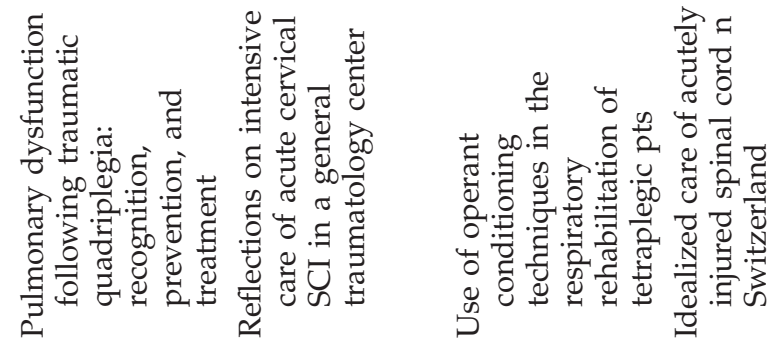

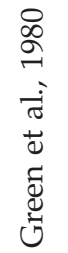

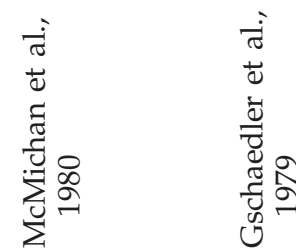

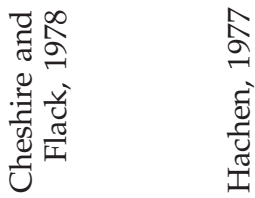




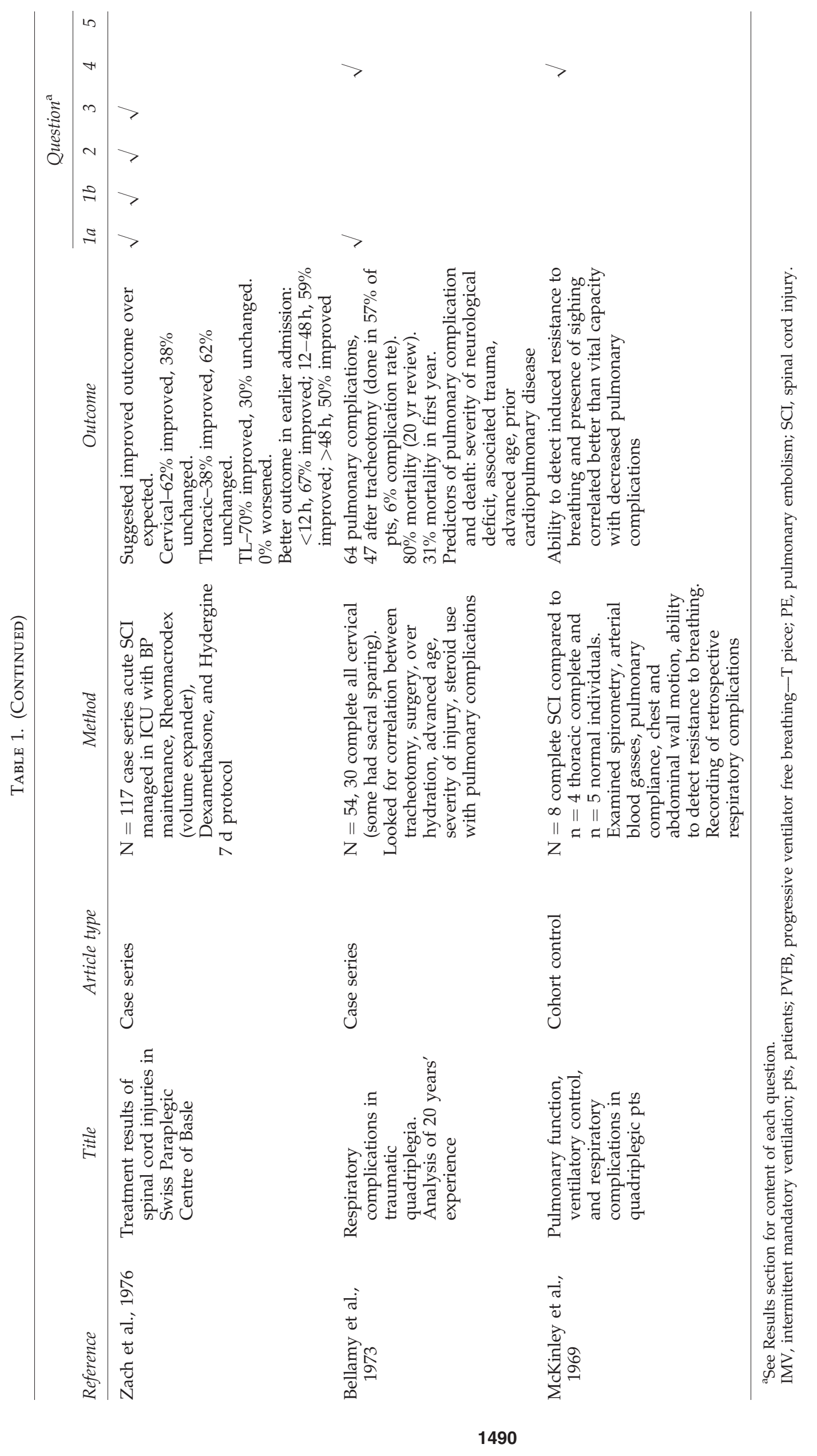


enced pulmonary complications in the first month including atelectasis, pneumonia, edema, pulmonary embolism, and aspiration and that this accounted for $11 \%$ of the mortality. Piepmeier and co-workers (1985) observed that endotracheal suctioning often required for pulmonary toilette in SCI patients (especially cervical SCI) was associated with severe bradycardia and cardiac arrest in $11 \%$ of patients within the first 2 weeks of injury. Ledsome and colleagues (1981) undertook serial pulmonary function tests in a group of acute cervical SCI patients. They observed vital capacity at $30 \%$ of predicted with C56 injury. This was worse if the injury was at C4 or above. Hypoxia was frequently seen even in the presence of adequate ventilation and normocarbia likely due to V/Q mismatch. Hachen (1997) found that most early deaths were due to pulmonary complications and that respiratory insufficiency occurred in $70 \%$ of cervical complete injured patients and $26.8 \%$ of those with incomplete injury. Similarly, a multicenter prospective case series examining the incidence of respiratory complications after acute SCI found that $67 \%$ experienced respiratory complications and $22.6 \%$ experienced respiratory failure (Jackson and Groomes, 1994). Respiratory complication rates were highest with C1-4 SCI $(84 \%)$ but were also frequent in lower cervical and thoracic SCI (60 and 65\%, respectively). Cervical SCI was also associated with hypoxia in $28 \%$ at admission in another study and $80 \%$ required ventilation (Myllynen et al., 1989). Again level and completeness of the injury were associated with a higher likelihood of respirator complication as were age, previous disease, and tachypnea at admission. Gardner and colleagues $(1985,1986)$ in reviewing patients who had required ventilator support found that $65 \%$ of ventilated SCI patients initiated it within $48 \mathrm{~h}$ and $43 \%$ died. Bain and colleagues (1995) compared imaging with chest x-ray and CT scan in SCI patients. They found that the chest CT scan was normal in only $20 \%$ of the patients examined and that $65 \%$ of cervical SCI patients without chest trauma exhibited lung changes. Similarly, a retrospective review of radiologically diagnosed pulmonary complications in acute tetraplegic patients found that $28 \%$ of patients exhibited lesions and that this was more frequent with complete injury $(40 \%)$ (Scher, 1982). Lemons and Wagner (1994) also reviewed the respiratory complications after acute SCI and found a $62 \%$ incidence, with $49 \%$ requiring ventilatory support. Bellamy and colleagues (1973) found that $57 \%$ of patients required tracheotomy. Pulmonary complications were associated with more severe neurological deficit, trauma, advanced age, and prior cardiopulmonary disease. Biering-Sørensen and Biering-Sørensen (1992) found that $11 \%$ of all spinal injured patients (including lumbar) required tracheotomy and that this was associated with cervical injury, more severe Frankel grade, and increased mortality. Thus, similar to the incidence of cardiovascular complications, many clinical series have documented a high rate of pulmonary complications, and specifically pulmonary insufficiency requiring ventilatory support. Specific factors associated with an increased risk of significant respiratory failure include high cervical (above C5) injury, increased injury severity (especially ASIA A injury), advanced age, prior respiratory disease, trauma, and tachypnea at admission.

Taken together, this literature confirms that SCI patients are at high risk for cardiovascular and pulmonary complications and often require intensive care related to those com- plications. Several authors have applied specific protocols to manage these complications in this patient population in the hopes of improving outcome. We found 21 articles with this objective, 15 case series, five cohort control studies, and one randomized control trial.

Several articles have defined a combination of surgical and medical management, often in a specialized center, and examined outcomes with these protocols. These approaches addressed intensive care with cardiovascular and pulmonary support. Vale and colleagues (1997) treated patients in order to maintain MAP $>85$ for 7 days using a protocol of colloid, blood transfusion, dopamine, and Levophed. They documented the outcome with this treatment as improvement of $>1$ Frankel or ASIA grade, as well as rate of ambulation. These data are summarized in Table 1 and are remarkable for the high rate of ambulation, even with cervical complete SCI $(20 \%)$. This was an uncontrolled study; however the results appear significantly better that those observed prior in the absence of the standardized approach applied by this group. Botel and co-workers (1997) undertook demonstrating the feasibility of admission to a SCI specialized unit within $24 \mathrm{~h}$ and in the process examined outcome. They found no improvement in grade occurred in Frankel A patients but found improvement in all other grades. Mortality was $8.6 \%$. The authors could not make any conclusion regarding the effect of treatment on outcome; however they indicated that prompt transfer to a specialized center was justified in these patients, given the high incidence of poly-trauma and other disease. Levi and co-workers (1993) treated acute SCI patients with maintenance of MAP >90 using fluids, dopamine, and dobutamine in an uncontrolled case series. They found that their protocol was feasible and safe and reduced morbidity and mortality. They observed complete injured patients (Frankel A or B) showed no neurological improvement when exhibiting persistent severe hemodynamic compromise (based on measurement of systemic and pulmonary vascular resistance using a Swan-Ganz catheter) whereas similar patients lacking severe compromise showed improvement. Furthermore, and distinct from other studies, these authors found that hemodynamic parameters did not differ between complete and incomplete SCI patients. Wolf and colleagues (1991) also included aggressive ICU management with MAP $>85$ in their patients with bilateral jumped facets and SCI. They observed neurological improvement in $21 \%$ of complete injured and $62 \%$ of incomplete injured patients at discharge, with sustained improvements at 1 year. They concluded that early reduction of the dislocation and aggressive ICU management, particularly targeted at MAP, improved outcome and reduced secondary complications. Tator and co-workers (1984) also admitted a cohort of patients to a specialized ICU addressing hypotension with crystalloid and whole blood infusions, as well as management of respiratory failure, and compared the group to a historical cohort treated without ICU care. They demonstrated that $43 \%$ were improved, $55 \%$ unchanged, $2 \%$ worse in the ICU cohort. They attributed improved neurological outcome and lower mortality and complications to their cardiopulmonary support protocol. They also observed decreased acute center length of stay in that cohort. Both Gschaedler and associates (1979) and Hachen (1977) also demonstrated decreased morbidity and mortality and suggested improved neurological outcome in a series of patients treated in the ICU with avoidance of hypotension and 
respiratory insufficiency. Zach and associates (1976) added rheomacrodex (a volume expander) to their 7 day protocol of blood pressure maintenance and observed neurological outcomes greater than expected, especially when patients were admitted early. McMichan and co-workers (1980) found that undertaking a protocol intended to prevent or reverse retention of pulmonary secretions in the ICU setting decreased mortality, pulmonary complications, intubation, and ventilation compared to a historical control cohort. This series of articles supports the hypothesis that neurological outcome is improved and morbidity and mortality are reduced with management of SCI patients in specialized centers with standardized intensive care management. Some articles included historical controls or demonstrated trends related to the timing of intervention or to the severity of the hemodynamic compromise to demonstrate improved recovery with this approach.

Thus, overall, the data that have been collected largely from case series but also from some cohort control studies have provided evidence that SCI patients are at significant risk of cardiovascular and respiratory insufficiency and that neurological outcome, complication rates, and mortality rates can be improved by management in specialized centers with access to intensive care facilities often with specific protocols established for the management of SCI.

In light of the significant incidence of cardiorespiratory complications, we conclude that SCI patients should be managed in a monitored special care unit with appropriate intensive ventilatory and cardiopulmonary support capability.

\section{What is the time at risk for cardiopulmonary complications requiring ICU management availability?}

Spinal cord injured patients often require a long hospital stay as they progress from acute management to rehabilitation and finally to more independent living. The preceding section examined the rationale for management in specialized centers; however it can be anticipated that the intensive facilities of those centers would be required in the acute phase with less of those resources required over time. It would therefore be useful to define the period at risk following SCI when ICU management may be required. We found 19 articles that provided some definition of this time period. These included 12 case series, six cohort control studies, and one randomized control trial.

Several of the articles that describe a protocol of cardiovascular and pulmonary intensive management in the acute phase also defined a period of time when that protocol was applied. The majority of these concentrated on cardiovascular dysfunction and support. Vale and colleagues (1997) and Zach and colleagues (1976) employed their treatment for 7 days while Wolf and colleagues (1991) applied it for 5 days. Zach and colleagues (1976) further demonstrated a correlation with improved outcome with earlier initiation of therapy. The time period of intervention was more rigorously defined by Levi and colleagues (1993), who applied fluid and inotropic support based on measurements made using a Swan-Ganz catheter. They observed duration of inotropic support of 5.4 days (required in $81 \%$ of patients). While the mean time of initiation of therapy was not explicitly defined in that study, the discussion implied that the first $24 \mathrm{~h}$ were critical in determining hemodynamic instability and prognosis. Botel and colleagues (1997) attempted to admit patients to a specialized unit within $24 \mathrm{~h}$ and were able to do so in $80 \%$ of cases. Lehmannn and colleagues (1987) studied a series of patients admitted to the ICU within $12 \mathrm{~h}$. They concentrated largely on cardiovascular events related to disturbance in sympathetic function and found that the period at risk was the first 14 days. They further commented that if these complications occurred, they resolved within 2 to 6 weeks. Piepmeier and colleagues (1985) similarly observed that endotracheal suctioning was associated with severe bradycardia and cardiac arrest within 2 weeks, but with regard to hypotension patients were most vulnerable during the first week.

Several articles examined more specifically pulmonary complications and the need for ventilatory support. Ledsome and Sharp (1981) serially followed pulmonary function in a series of SCI patients and observed a significant improvement in vital capacity within the first 5 weeks of injury with doubling by 3 months. Jackson and Gromes (1994) examined the incidence of respiratory complications and found that ventilatory failure occurred at mean 4.5 days and had a mean duration of 35.9 days. Pneumonia and atelectasis tended to present in a more delayed fashion. Lu and colleagues (2000) identified a cohort of patients that experienced delayed apnea between days 9 and 15 following a period of clinical stability, also emphasizing that pulmonary complications may be more delayed. Gardner and colleagues (1986) found that $66 \%$ of patients that required ventilation did so within $48 \mathrm{~h}$ and that $19 \%$ required greater that 8 weeks of ventilation. Those able to start ventilatory weaning earlier also were able to wean faster. Lemons and Wagner (1994) found severity of cervical SCI was a predictor of ventilation and ICU length of stay. Cervical complete injured patients required a mean 30 days of ICU management. Overall $49 \%$ of patients required ventilation.

This literature suggests that the period of cardiovascular instability, which is often due to sympathetic dysfunction, is most pronounced acutely and that a period at risk of between 1 and 2 weeks exists. Respiratory insufficiency however tends to be more prolonged with ventilation when required, usually occurring within the first few days but not infrequently lasting for weeks. Delayed pulmonary complications must also be anticipated. Thus, the period at risk for intensive management of SCI patients appears to start very early if not immediately following the injury but may extend beyond a month due to prolonged ventilatory requirements that are frequently encountered.

\section{What parameters for blood pressure and oxygenation/ventilation over what time period are associated with improved outcome?}

The literature defining the appropriate physiological parameters that should be used in the intensive care management of SCI patients is somewhat smaller than that defining the need for such management. We identified eight articles that specified management parameters, six case series, and two cohort control studies.

Vale and colleagues (1997) administered methylprednisolone and a hemodynamic management protocol using colloid, blood, dopamine, and Levophed aiming to maintain MAP $>85 \mathrm{mmHg}$ for 7 days. Levi and colleagues (1993) similarly used fluids, dopamine, and dobutamine to maintain MAP $>90 \mathrm{mmHg}$. Wolf and colleagues (1991) aimed for MAP > 85 for 5 days. Piepmeier and colleagues (1985) used vasopres- 
sors to maintain SBp $>100$. These studies have in common the selection of a target MAP to guide therapy (Levi and co-workers [1993] also used other hemodynamic parameters). The strength of evidence that these interventions impacted outcome is described above. We did not find any articles where optimization of parameters such as MAP was undertaken. Interestingly all aim at maintaining MAP around 85 or $90 \mathrm{mmHg}$. None of the identified articles provided details of the ventilatory management applied in the acute setting.

We conclude therefore that this literature supports a recommendation, based on weak evidence, that MAP >85 $\mathrm{mmHg}$ be maintained following SCI for a period extending up to 1 week following injury.

\section{Are there any risk factors that are predictive of the need for ICU management?}

While generally SCI patients are a high-risk group in terms of complications and adverse events, they do not represent a homogeneous population. It is intuitive that there are subgroups of patients where the index of suspicion for such events should be higher. In an attempt to identify predictors that would identify these subgroups we selected those articles that described variations in the need for intensive care management by patient characteristics. We identified 19 articles; 13 were case series, four were cohort control studies, and two were evaluations of a diagnostic procedure.

The most frequently examined predictors of the need for both cardiovascular and pulmonary support were injury level and severity of injury.

Vale and colleagues (1997) found that, using their protocol aimed at maintaining MAP $>85,90 \%$ of cervical-complete patients required pressors while $52 \%$ of incomplete and $31 \%$ of thoracic require pressors. Similarly, Lehmann and colleagues (1987) demonstrated that patients with severe cervical SCI experienced more cardiovascular complications (bradycardia, hypotension, arrhythmia, cardiac arrest, and need for pressors) than did those with less severe cervical SCI and in turn those with thoracolumbar SCI. Severity of SCI was also correlated with the incidence and severity of cardiovascular irregularity by Piepmeier and colleagues (1985).

Reines and Harris (1987) demonstrated increased incidence of pneumonia, atelectasis, and death due to pulmonary complications in those patients with higher cervical SCI. Ledsome and Sharp (1981) found that the reduced vital capacity and peak flow rate seen after SCI was worse if C4 was involved in the injury. Similarly, Myllynen and colleagues (1989) found that level of injury and completeness of injury were predictive of the need for ventilation. Scher and colleagues (1982) found that pulmonary lesions were more frequently seen on chest imaging of complete injured SCI patients than of incomplete injured patients. In examining respiratory complications after SCI, Lemons and Wagner (1994) found that severity of injury was predictive of ventilation and length of ICU stay but that level of injury failed to correlate significantly with incidence or severity of respiratory disease. Biering-Sørensen and Biering-Sørensen (1992) found that both cervical level and Frankel grade (severity) were predictive of the need for tracheotomy.

Other risk factors, particularly for pulmonary complications and the need for ventilation, have been identified. Myllynen and colleagues (1989) found the respiratory rate at admission (tachypnea), increased age, and previous respiratory disease correlated with the occurrence of pulmonary complications. Bellamy and colleagues (1973) similarly identified advanced age, prior cardiopulmonary disease, and associated trauma, in addition to severity of the neurological deficit, were predictive of pulmonary compilations and death. Claxton and colleagues (1998) using both univariate and multivariate analyses implicated SCI above the C4 level, copious sputum at 1 week, pneumonia, and major lobe collapse in predicting the need for ventilatory support. Lu and colleagues (2000) examined risk factors associate with the development of delayed apnea and found that a diffuse spinal cord lesion, involvement of the C4 spinal level, and clinical events including bradycardia, hypotension, or dyspnea (typically days prior to apnea) correlated with the occurrence of apnea. Lesoin and colleagues (1983) used diaphragm EMG to examine ventilated patients after SCI and found that, of seven patients that failed to demonstrate EMG, 6 died and one failed to wean. Roth and colleagues (1997) suggested that muscle strength was more important than tone in determining pulmonary function although both exhibited a role. Piepmeier and colleagues (1985) found that endotracheal suctioning was associated with severe bradycardia and cardiac arrest within the first 2 weeks, suggesting that difficulty with pulmonary toilette is a risk factor for these cardiovascular events.

Thus many predictors of poor cardiovascular and respiratory functioning requiring resuscitation and support have been identified. The predictors of cardiovascular complications are high cervical and complete SCI. While both of these as well as lack of diaphragm function, advanced age, previous cardiopulmonary disease, tachypnea at admission, copious sputum, pneumonia, and major lobe collapse are predictive of the need for ventilatory support.

\section{What preventative strategies (excluding DVT prophylaxis, surgical management, and nutrition) can be undertaken to reduce the risks of cardiopulmonary complications requiring ICU care?}

The preceding results confirm that SCI patients are at high risk for cardiovascular and respiratory complications often requiring intensive management. Furthermore, some predictive risk factors for these adverse events have been identified. Some of those risk factors may be modifiable, thus impacting the risk. In this regard we identified nine articles in our search that described strategies to decrease the risks of cardiac or pulmonary complications requiring ICU care or decrease the length of ICU management. These included five case series, three cohort control studies, and one randomized control trial.

All the articles identified attempted to reduce the incidence of pulmonary complications. Several articles addressed the issue of impaired secretions and atelectasis. In a cohort with retrospective control study, McMichan and colleagues (1980) demonstrated that a protocol intended to prevent or reduce pulmonary secretions in the ICU decreased mortality, pulmonary complications, and the need for mechanical ventilation. Cheshire and Flack (1978) similarly undertook an incentive spirometry program and demonstrated that with this strategy large improvements in vital capacity could be achieved although this study lacked a control arm. Sugarman (1985) undertook vigorous pulmonary therapy to prevent and treat atelectasis and found that this resulted in prompt 
normalization of chest $\mathrm{x}$-ray findings and clinical improvement. Lemons and Wagner (1994) compared their standard respiratory protocol to one that added a rotating bed and chest percussion therapy and found that the later group displayed less pneumonias and shorter ICU stays.

Other articles addressed weaning or respiratory support to decrease the need for ventilation. Tromans and colleagues (1998) applied BiPAP on the ward to prevent respiratory failure or to wean off ventilation. Derrickson and colleagues (1992) compared abdominal weights to inspiratory resistive muscle training in a small, randomized trial. Both groups showed improvement in spirometry over time but no differences between the groups was demonstrated. Peterson and colleagues (1994) compared ventilator weaning using IMV (intermittent mandatory ventilation) and progressive ventilatory-free breathing and demonstrated some advantage to the latter.

Finally two articles explored the use of specific beds to nurse SCI patients. Borkowski (1989) compared a kinetic treatment table that placed patients in perpetual motion to a wedge turning device that allowed for single person assisted turning without lifting and showed an advantage to the former in reducing need for intubation, tracheotomy, pulmonary infections, and length of time on ventilation. Green and coworkers (1980) also examined a bed allowing perpetual motion and reported reduction in a broad range of complications including respiratory and cardiovascular complications.

Thus, several attempts have been made to reduce pulmonary complications or to hasten resolution of pulmonary complications. From review of this literature, atelectasis and copious secretions stand out as frequent obstacles to ventilator weaning and discharge from the ICU. Strategies to reduce or promptly treat these appear to have met with some success although the evidence comes from largely uncontrolled studies or studies that compare to a retrospective cohort. Thus, there is weak evidence to support a recommendation to address copious secretions through chest physiotherapy and suctioning in order to avoid further respiratory complications.

\section{Discussion}

The patient with SCI frequently presents several clinical challenges, many of which are not well informed by the literature. For example, controversies remain regarding the timing of surgery in some patients and discussion regarding the role of steroids (such as methylprednisolone) continues. In this review we have concentrated on the intensive care of such patients and have attempted to address several questions as described in the Results section. These questions will not be reiterated here as they are each discussed in detail above. We generally found that a significant amount of literature exists demonstrating the high incidence of cardiopulmonary complications encountered by these patients and that several studies suggest improved clinical outcomes when those complications are the subject of focused management strategies in specialized centers. However, there are few controlled studies in this area, an observation that is not surprising since cardiopulmonary resuscitation is a basic principal of trauma management and randomization of critically ill patients to resuscitation versus observation or intensive treatment versus non-specialized care would be unethical. Thus, while much of the literature in this area is comprised of case series with some cohort control studies, it is unlikely that some of the fundamental questions posed in this review will be addressed by high-level evidence in the future. Nonetheless, the literature lacks attempts at optimizing the intensive management of the SCI patient, an area that could be subject to further scrutiny. For example, while the current literature is supportive of the need for prompt management in specialized units with access to the ICU and suggests that maintenance of MAP $>85$ is a key ingredient in the management approach, none have attempted to optimize this hemodynamic target. It would seem that the target MAP and the agents used to achieve it may be subject to retrospective observational studies examining the success of hemodynamic resuscitation and its implications for outcome, or preferably could be subject to randomized studies examining variations of the target MAP or the specific agents used to achieve it.

While more work of this nature could further optimize the management of the patient with SCI, the observations made in our review, when scrutinized through a Delphi process, have led to two specific recommendations stemming from the questions posed. These are that, in light of the significant incidence of cardiorespiratory complications, patients with SCI should be managed in a monitored special care unit; there is weak evidence supporting the maintenance of MAP $>85 \mathrm{mmHg}$ for a period extending up to one week following SCI.

\section{Author Disclosure Statement}

No competing financial interests exist.

\section{References}

Bain, G., Bodley, R., Jamous, A., Williams, S., and Silver, J. (1995). A comparison of the chest radiograph and computerised tomography in assessing lung changes in acute spinal injuries-an assessment of their prevalence and the accuracy of the chest X-ray compared with CT in their assessment. Paraplegia 33, 121-125.

Bellamy, R., Pitts, F.W., and Stauffer, E.S. (1973). Respiratory complications in traumatic quadriplegia. Analysis of 20 years' experience. J. Neurosurg. 39, 596-600.

Biering-Sørensen, M., and Biering-Sørensen, F. (1992). Tracheostomy in spinal cord injured: frequency and follow up. Paraplegia 30, 656-660.

Borkowski, C. (1989). A comparison of pulmonary complications in spinal cord-injured patients treated with two modes of spinal immobilization. J. Neurosci. Nurs J. 21, 79-85.

Botel, U., Glaser, E., and Niedeggen, A. (1997). The surgical treatment of acute spinal paralysed patients. Spinal Cord 35, 420-428.

Cheshire, D.J., and Flack, W.J. (1978). The use of operant conditioning techniques in the respiratory rehabilitation of the tetraplegic. Paraplegia 16, 162-174.

Claxton, A.R., Wong, D.T., Chung, F., and Fehlings, M.G. (1998). Predictors of hospital mortality and mechanical ventilation in patients with cervical spinal cord injury. Can. J. Anaesth. 45, 144-149.

Derrickson, J., Ciesla, N., Simpson, N., and Imle, P.C. (1992). A comparison of two breathing exercise programs for patients with quadriplegia. Phys. Ther. 72, 763-769.

DeVivo, M.J., and Ivie, C.S. (1995). Life expectancy of ventilatordependent persons with spinal cord injuries. Chest 108, 226-232. 
Fuhrer, M.J., Carter, R.E., Donovan, W.H., Ross,i C.D., and Wilkerson, M.A. (1987). Postdischarge outcomes for ventilator-dependent quadriplegics. Arch. Phys. Med. Rehab. 68, 353-356.

Gardner, B.P., Theocleous, F., Watt, J.W., and Krishnan, K.R. (1985). Ventilation or dignified death for patients with high tetraplegia. Br. Med. J. 291, 1620-1622.

Gardner, B.P., Watt, J.W., and Krishnan, K.R. (1986). The artificial ventilation of acute spinal cord damaged patients: a retrospective study of forty-four patients. Paraplegia 24,208220.

Green, B.A., Green, K.L., and Klose, K.J. (1980). Kinetic nursing for acute spinal cord injury patients. Paraplegia 18, 181-186.

Gschaedler R., Dollfus, P., Mole, J.P., Mole, L., and Loeb, J.P. (1979). Reflections on the intensive care of acute cervical spinal cord injuries in a general traumatology centre. Paraplegia 17, 58-61.

Hachen, H.J. (1977). Idealized care of the acutely injured spinal cord in Switzerland. J. Trauma 17, 931-936.

Jackson, A.B., and Groomes, T.E. (1994). Incidence of respiratory complications following spinal cord injury. Arch. Phys. Med. Rehab. 75, 270-275.

Ledsome, J.R., and Sharp, J.M. (1981). Pulmonary function in acute cervical cord injury. Am. Rev. Respir. Dis. 124, 41-44.

Lehmann, K.G., Lane, J.G., Piepmeier, J.M., and Batsford, W.P. (1987). Cardiovascular abnormalities accompanying acute spinal cord injury in humans: incidence, time course and severity. J. Am. Coll. Cardiol. 10, 46-52.

Lemons, V.R., and Wagner, F.C. (1994). Respiratory complications after cervical spinal cord injury. Spine 19, 2315-2320.

Lesoin, F., Delandsheer, E., Lozes, G., Hurtevent, J.F., Jomin, M., and Guieu, J.D. (1983). Electromyography of the diaphragm in patients with cervical spine lesions necessitating assisted ventilation. Lancet 1, 1385-1386.

Levi, L., Wolf, A., and Belzberg, H. (1993). Hemodynamic parameters in patients with acute cervical cord trauma: description, intervention, and prediction of outcome. Neurosurgery 33, 1007-1016; discussion, 1016-1007.

Lu, K., Lee, T.C., Liang, C.L., and Chen, H.J. (2000). Delayed apnea in patients with mid to lower cervical spinal cord injury. Spine 25, 1332-1338.

McKinley, A.C., Auchincloss, J.H., Gilbert, R., and Nicholas, J.J. (1969). Pulmonary function, ventilatory control, and respiratory complications in quadriplegic subjects. Am. Rev. Respir. Dis. $100,526-532$.

McMichan, J.C., Michel, L., and Westbrook, P.R. (1980). Pulmonary dysfunction following traumatic quadriplegia. Recognition, prevention, and treatment. JAMA 243, 528-531.

Myllynen, P., Kivioja, A., Rokkanen, P., and Wilppula, E. (1989). Cervical spinal cord injury: the correlations of initial clinical features and blood gas analyses with early prognosis. Paraplegia 27, 19-26.
Peterson, W., Charlifue, W., Gerhart, A., and Whiteneck, G. (1994). Two methods of weaning persons with quadriplegia from mechanical ventilators. Paraplegia 32, 98-103.

Peterson, W.P., Barbalata, L., Brooks, C.A., Gerhart, K.A., Mellick, D.C., and Whiteneck, G.G. (1999). The effect of tidal volumes on the time to wean persons with high tetraplegia from ventilators. Spinal Cord 37, 284-288.

Piepmeier, J.M., Lehmann, K.B., and Lane, J.G. (1985). Cardiovascular instability following acute cervical spinal cord trauma. Cent. Nerv. Syst. Trauma 2, 153-160.

Reines, H.D., and Harris, R.C. (1987). Pulmonary complications of acute spinal cord injuries. Neurosurgery 21, 193-196.

Roth, E.J., Lu, A., Primack, S., Oken, J., Nusshaum, S., Berkowitz, M., and Powley, S. (1997). Ventilatory function in cervical and high thoracic spinal cord injury. Relationship to level of injury and tone. Am. J Phys. Med. Rehab. 76, 262-267.

Scher, A.T. (1982). The radiology of pulmonary complications associated with acute spinal cord injury. S. Afr. Med J. 62, 321-324.

Sugarman, B. (1985). Atelectasis in spinal cord injured people after initial medical stabilization. J. Am. Paraplegia Soc. 8, 47-50.

Tator, C.H., Rowed, D.W., Schwartz, M.L., Gertzbein, S.D., Bharatwal, N., Barkin, M., and Edmonds, V.E. (1984). Management of acute spinal cord injuries. Can. J. Surg. 27, 289-293, 296.

Tromans, A.M., Mecci, M., Barrett, F.H., Ward, T.A., and Grundy, D.J. (1998). The use of the BiPAP biphasic positive airway pressure system in acute spinal cord injury. Spinal Cord 36, 481-484.

Vale, F.L., Burns, J., Jackson, A.B., and Hadley, M.N. (1997). Combined medical and surgical treatment after acute spinal cord injury: results of a prospective pilot study to assess the merits of aggressive medical resuscitation and blood pressure management. J. Neurosurg. 87, 239-246.

Vogel, L.C. (1997). Unique management needs of pediatric spinal cord injury patients: medical issues. J. Spinal Cord Med. 20, 17-20.

Wolf, A., Levi, L., Mirvis, S., Ragheb, J., Huhn, S., Rigamonti, D., and Robinson, W.L. (1991). Operative management of bilateral facet dislocation. J. Neurosurg. 75, 883-890.

Zach, G.A., Seiler, W., and Dollfus, P. (1976). Treatment results of spinal cord injuries in the Swiss Parplegic Centre of Basle. Paraplegia 14, 58-65.

Address correspondence to: Steven Casha, M.D., Ph.D. QEII Health Sciences Centre Halifax Infirmary

1796 Summer Street, Room 3808 Halifax, Nova Scotia Canada B3H $3 A 7$

E-mail: scasha@dal.ca 
